\title{
Strategies for the Management of Early-Stage Breast Cancer in Older Women
}

Presented by Lee S. Schwartzberg, MD, and Sarah L. Blair, MD

\begin{abstract}
Older patients with breast cancer (aged $\geq 65$ years) are often undertreated with both locoregional and systemic therapies and have been shown to have higher breast cancer-specific mortality. These patients are also excluded from most clinical trials; therefore, treatment recommendations are extrapolated from younger populations. The data that do exist, however, show that older patients usually tolerate and respond well to conventional treatments. When selecting treatments for breast cancer, age should not be the chief consideration; comorbidities and functional status are also important, as is life expectancy. For patients with an estimated survival of less than 5 years, aggressive treatment may be discouraged; however, if the estimated survival is 5 years or more, treatment according to recurrence risk is recommended. In the curative setting, undertreatment should be avoided.
\end{abstract}

J Natl Compr Canc Netw 2016;14(5.5):647-650

\section{Undertreatment of Early-Stage Breast Cancer in Older Women}

Many elderly women (aged $\geq 65$ years) with early-stage breast cancer are candidates for standard treatments, yet they often do not receive them, according to 2 members of the NCCN Guidelines Panel for Breast Cancer who described this population and made recommendations at the NCCN 21st Annual Conference.

"The paradox is that older women generally have more favorable [tumor] biology and a less advanced stage at diagnosis, but they have worse breast cancer-specific mortality. Why is that?" questioned Lee S. Schwartzberg, MD, Executive Director of West Cancer Center,

\footnotetext{
Presented by Lee S. Schwartzberg, MD, The University of Tennessee Health Science Center, West Cancer Center, Memphis, Tennessee, and Sarah L. Blair, MD, Department of Surgery, University of California San Diego Moores Cancer Center, La Jolla, California.

Dr. Schwartzberg has disclosed that he receives consulting fees and/ or honoraria from Amgen Inc., Genentech, Inc., and TESARO, Inc.; is a scientific advisor for Bristol-Myers Squibb Company, Caris Life Sciences, and Helsinn; and is on the product/speakers bureau for Genentech, Inc. and Pfizer Inc. Dr. Blair has disclosed that she has no financial interests, arrangements, affiliations, or commercial interests with the manufacturers of any products discussed in this article or their competitors.

Correspondence: Lee S. Schwartzberg, MD, The University of Tennessee Health Science Center, West Cancer Center, Hematology/Oncology, 7945 Wolf River Boulevard, Germantown, TN 38138.

E-mail: Ischwartzberg@westclinic.com

Sarah L. Blair, MD, Department of Surgery, University of California San Diego Moores Cancer Center, 3855 Health Science Drive, La Jolla, CA 92093. E-mail: slblair@ucsd.edu
}

Professor of Medicine and Chief, Division of Hematology/Oncology at The University of Tennessee Health Science Center. The reasons for this, he believes, include undertreatment, inadequate data from clinical trials, and potentially age-related reduced immune surveillance.

When selecting treatment for older patients, he suggested, "Don't make decisions based on age alone, but also consider the patient's goals, life expectancy, and totality of morbidities." The mandate is to avoid both undertreatment in the curative setting and toxicity in the palliative setting.

"With proper support, standard therapies are tolerable. We can get patients through treatments without losing their independence or having permanent declines in function," Dr. Schwartzberg said.

\section{Older Patients at Higher Risk}

Older patients with breast cancer are a rapidly growing component of oncology practice. With a median age at breast cancer diagnosis of 61 years, more than $40 \%$ of patients are diagnosed after 65 years and $21 \%$ after 75 years. Although the mortality risk from breast cancer has been declining in general, among the subset of patients older than 70 years with estrogen receptor (ER)negative disease, almost no increase in survival has been observed. ${ }^{1}$ 
Furthermore, breast cancer-specific mortality risk increases with age, according to Sarah L. Blair, MD, Clinical Professor in the Department of Surgery at the University of California, San Diego. Dr. Blair's analysis of SEER data showed that elderly patients with hormone receptor (HR)-negative disease are more likely to be inadequately treated and to die of breast cancer than their younger counterparts, and are more likely to die of breast cancer than of cardiovascular disease. ${ }^{2}$

"Compared with women in their 50s and 60s, those older than 80 years were less likely to have surgery and radiotherapy [RT], and their overall survival was worse," she said. These patients are also less likely to undergo screening for breast cancer, despite a 3-fold increase in risk compared with younger patients. ${ }^{3}$ The US Preventive Services Task Force recommends that mammography screening be stopped at age 74 years, although the most recent guidelines from the American Cancer Society recommend discontinuing screening when a woman's life expectancy is less than 10 years, she said.

\section{Inadequate Locoregional Treatment}

Several large studies have noted the inadequate locoregional treatment of older patients. Standard surgical treatment includes lumpectomy plus axillary staging and RT, or mastectomy plus axillary staging, Dr. Blair reminded the audience.

In a Swiss population study of 4,820 elderly patients with breast cancer, women older than 80 years were less likely to undergo staging, which translated to a higher recurrence rate of 4\% (no axillary staging) compared with $1 \%$ (with axillary staging) in younger patients. Additionally, after breast-conserving surgery, elderly patients were less likely to have adjuvant $\mathrm{RT}$ and chemotherapy. ${ }^{4}$ Similarly, in a recent Canadian study, axillary staging was less frequent among women 80 years of age and older, ${ }^{5}$ and in a 2013 study, omission of surgery was associated with worse-than-expected breast cancer-specific mortality. ${ }^{6}$ In another study, breast cancer-specific survival among octogenarians was significantly lower for patients receiving suboptimal surgical treatment. ${ }^{7}$

In "the real world," said Dr. Blair, older patients do sometimes choose less aggressive treatments, "but this does have its cost." A SEER database analysis of 1,837 elderly patients with stage I or II disease found breast cancer mortality to be twice as high for those receiving breast-conserving surgery without RT versus mastectomy, and 6 times higher for those receiving tamoxifen for less than 1 year compared with 5 years or more. ${ }^{8}$ However, based on a prospective randomized clinical trial, carefully selected patients with small hormone-sensitive tumors and who have been compliant with endocrine therapy for 5 years are able to have breast conservation without radiation and preserve overall survival. ${ }^{9}$

\section{Determining Systemic Treatment for Older Patients}

Systemic treatment is often inadequate in older patients, a topic discussed at the NCCN Annual Conference by Dr. Schwartzberg. Clinicians should always consider the patient's treatment goals, functional status, and life expectancy, he emphasized, noting that most healthy 80 -year-olds now live to be approximately 90 years of age.

However, especially in the frail population, there is a need to avoid further loss of function or independence. For older, retired patients, financial toxicity may also be an issue, he added. When deciding how aggressively to treat the cancer, Dr. Schwartzberg suggested that clinicians use a geriatric assessment tool, online treatment planning tools, and the 21-gene recurrence score (RS) assay. A 2015 study of the Medicare population showed that use of the 21-gene RS assay in this population was quite low, at only $15 \%$ among patients aged 76 to 80 years. ${ }^{10}$ "This suggests that perhaps these patients are not even being considered for chemotherapy, and the genomic profile is not used because the decision was made a priori," he said.

The benefit of the RS in older patients was recently confirmed in a study showing that recurrence rates lined up with RS among patients aged 70 years and older, and especially among those older than 80 years. Interestingly, despite a high RS, older patients were far less likely to receive chemotherapy than younger patients. ${ }^{11}$

\section{Benefit of Endocrine Therapy}

The benefit of aromatase inhibitors over tamoxifen extends to both older and younger patients, according to the Early Breast Cancer Trialists' Collaborative Group meta-analysis. ${ }^{12}$ Furthermore, in the MA.17 study, an additional 5 years of letrozole after 
Early-Stage Breast Cancer in Older Women

\begin{tabular}{|llll|}
\hline \multirow{2}{*}{ HER2- } & Regimen & Evidence in Elderly & Recommendations \\
\cline { 2 - 4 } & AC->T & Subgroup & Only fit and high-risk pts \\
& CMF & Prospective & Feasible with precautions \\
& AC & Prospective & Feasible with precautions \\
& TC & Subgroup & Feasible with G-CSF \\
& Weekly doc & Prospective & Not recommended \\
& Weekly pac & Subgroup & Feasible \\
\hline HER2+ & AC->TH & Subgroup & Only fit and high-risk pts \\
& TCH & Minimal & Only fit and high-risk pts \\
& Weekly Pac-H & Subgroup & Feasible \\
& TC-H & No evidence & Feasible \\
\hline
\end{tabular}

Figure 1. Decision-making for chemotherapy in elderly women.

Abbreviations: AC, anthracycline/cyclophosphamide; CMF, cyclophosphamide/methotrexate/fluorouracil; doc, docetaxel; G-CSF, granulocyte colonystimulating factor; pts, patients; T, paclitaxel; TC, docetaxel/cyclophosphamide; TCH, docetaxel/carboplatin/trastuzumab.

5 years of tamoxifen reduced recurrences in patients aged 65 years and older. ${ }^{13}$

"Based on these data, for the 'younger' older woman completing 5 years of adjuvant therapy who is at risk for recurrence, an additional 5 years of treatment is warranted," according to Dr. Schwartzberg. He also recommended bone-targeting agents in older women with osteopenia or osteoporosis because of their bone-protective and antitumor effects in the estrogen-deprived population. ${ }^{14}$

\section{Choice of Chemotherapy in the Older Patient}

Certainly, many elderly patients can, or should, forego aggressive interventions. "In patients who are frail and already dependent, [clinicians] have to be cautious about using adjuvant therapy," he said. "And you could make the case that an 85-year-old with a $1-\mathrm{cm}$, strongly ER-positive, grade 1 tumor has a very low likelihood of breast cancer recurrence after adequate local therapy." However, for many patients, clinicians should choose the best chemotherapy regimen. In brief, Dr. Schwartzberg recommended estimating life expectancy without breast cancer and without chemotherapy, and basing treatment on expected incremental survival (Figure 1).

Although there is a "data void" for treatment recommendations in women older than 70 years, patients appear to derive a greater benefit from combination chemotherapy than from single agents-as with younger patients-and without much additional toxicity. Novel regimens have not improved on the "old standards," he added.
For HR-negative disease, 3 treatment regimens are recommended, all of which should be accompanied by growth factor support and careful organ function monitoring in patients aged 65 years and older:

- Dose-dense anthracycline/cyclophosphamide (AC) followed by paclitaxel

- Dose-dense AC followed by weekly paclitaxel

- Docetaxel/cyclophosphamide (TC)

For the $15 \%$ of patients with HER2-positive disease, the NCCN Guidelines for Breast Cancer include numerous regimens that combine chemotherapy with trastuzumab with and without pertuzumab. Of note, a careful assessment of cardiac risk should be performed in this patient population.

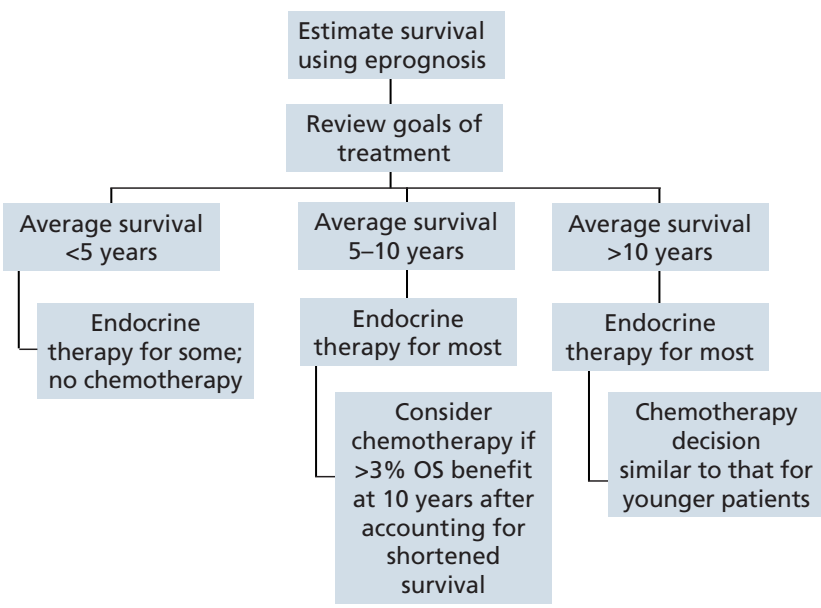

Figure 2. Recommendations for adjuvant chemotherapy in older patients with breast cancer.

Abbreviation: OS, overall survival. 
Although most clinicals trials exclude older women, $10 \%$ of patients in the recent APT trial were older than 70 years. In this study, patients with HER2-positive, node-negative small tumors received paclitaxel weekly plus trastuzumab weekly for 12 weeks, with trastuzumab continued for 1 year. ${ }^{15}$ Disease-free survival overall was an impressive $98.7 \%$ at 3 years. "This study provides us with good information on a program for treating the elderly with HER2-positive disease," he said.

Dr. Schwartzberg concluded with some succinct recommendations for treating older patients (Figure 2).

\section{References}

1. Jatoi I, Chen BE, Anderson WF, Rosenberg PS. Breast cancer mortality trends in the United States according to estrogen receptor status and age at diagnosis. J Clin Oncol 2007;25:1683-1690.

2. Weiss A, Noorbakhsh A, Tokin C, et al. Hormone receptor-negative breast cancer: undertreatment of patients over 80. Ann Surg Oncol 2013;20:3274-3278.

3. Kimick GG, Muss HB. Breast disease. In: Halter JB, Ouslander JG, Tinetti $\mathrm{ME}$, et al, eds. Hazzard's Geriatric Medicine and Gerontology. Columbus, OH: McGraw Hill; 2009

4. Joerger M, Thurlimann B, Savidan A, et al. Treatment of breast cancer in the elderly: a prospective population-based Swiss Study. J Geriatr Oncol 2013;4:39-47.
5. Angarita FA, Chesney T, Elser C, et al. Treatment patterns of elderly breast cancer patients at two Canadian cancer centres. Eur J Surg Oncol 2015;41:625-634.

6. Hamaker ME, Bastiaannet E, Evers D, et al. Omission of surgery in elderly patients with early stage breast cancer. Eur J Cancer 2013;49:545-552.

7. Cortadellas T, Gascón A, Córdoba $O$, et al. Surgery improves breast cancer-specific survival in octogenarians with early-stage breast cancer. Int J Surg 2013;11:554-557.

8. Yood MU, Owusu C, Buist DS, et al. Mortality impact of less-than-standard therapy in older breast cancer patients. J Am Coll Surg 2008;206:66-75.

9. Hughes KS, Schnaper LA, Bellon JR, et al. Lumpectomy plus tamoxifen with or without irradiation in women age 70 years or older with early breast cancer: long-term follow-up of CALGB 9343. J Clin Oncol 2013;31:23822387.

10. Dinan MA, Mi X, Reed SD, et al. Initial trends in the use of the 21-gene recurrence score assay for patients with breast cancer in the Medicare population, 2005-2009. JAMA Oncol 2015;1:158-166.

11. Shak S, Petkov VI, Miller DP, et al. Breast cancer specific survival in 38,568 patients with node-negative hormone receptor-positive invasive breast cancer and Oncotype DX recurrence score results in the SEER database [abstract]. Cancer Res 2016;76(4 Suppl):Abstract P5-15-01.

12. Early Breast Cancer Trialists' Collaborative Group. Aromatase inhibitors versus tamoxifen in early breast cancer: patient-level meta-analysis of the randomized trials. Lancet 2015;386:1341-1352.

13. Huss HB, Tu D, Ingle JN, et al. Efficacy, toxicity, and quality of life in older women with early-stage breast cancer treated with letrozole or placebo after 5 years of tamoxifen: NCIC CTG intergroup trial MA.17. J Clin Oncol 2008;26:1956-1964.

14. Early Breast Cancer Trialists' Collaborative Group (EBCTCG), Coleman $\mathrm{R}$, Powles T, et al. Adjuvant bisphosphonate treatment in early breast cancer: meta-analyses of individual patient data from randomized trials. Lancet 2015;386:1353-1361.

15. Tolaney SM, Barry WT, Dang CT, et al. Adjuvant paclitaxel and trastuzumab for node-negative, HER2-positive breast cancer. N Engl J Med 2015;372:134-141. 